PROCEEDINGS OF THE

AMERICAN MATHEMATICAL SOCIETY

Volume 132, Number 10, Pages 3019-3025

S 0002-9939(04)07333-2

Article electronically published on June 2, 2004

\title{
BICIRCULAR PROJECTIONS AND CHARACTERIZATION OF HILBERT SPACES
}

\author{
LÁSZLÓ L. STACHÓ AND BORUT ZALAR \\ (Communicated by David R. Larson)
}

\begin{abstract}
We prove that every JB* triple with rank one bicircular projection is a direct sum of two ideals, one of which is isometrically isomorphic to a Hilbert space.
\end{abstract}

\section{INTRODUCTION}

The topic, which we study in the present paper in a completely algebraic way, was motivated by our previous investigation of continuous Reinhardt domains. These are open subsets of $C_{0}(\Omega)$, satisfying some additional properties (cf. [18]). In [18] we were led, at some technical point, to study projections with one-dimensional range and additional norm property, which is called bicircularity. What we needed to show was that their kernels are Hilbert spaces.

The definition of bicircularity is however a general Banach space notion, which is not necessarily connected to $C_{0}(\Omega)$. We thus expand, what was a technical point in [18], into an independent study in its proper algebraic setting, which is that of JB* triples. Our main result and its corollary can be interpreted as a characterization of Hilbert spaces among JB* triples.

\section{Bicircular PROJECTIONS AND JB* TRIPLES}

Let $W$ be a complex Banach space and $P: W \rightarrow W$ a projection. We denote by $\bar{P}$ the projection $1-P$. Clearly, $P \bar{P}=\bar{P} P=0$ holds.

Definition 1. The projection $P$ is called bicircular if for all $\alpha, \beta \in R$ the operator $e^{i \alpha} P+e^{i \beta} \bar{P}$ is an isometry.

Every bicircular projection is automatically bicontractive, i.e., $\|P\| \leq 1$ and $\|\bar{P}\| \leq 1$. This follows from the above definition by taking $e^{i \alpha}=1$ and $e^{i \beta}=-1$. Then $\phi=P-(1-P)=2 P-1$ is isometric and so

$$
\|P\|=\frac{1}{2}\|1+\phi\| \leq \frac{1}{2}(1+\|\phi\|)=1 .
$$

In a similar way we can prove that $\|\bar{P}\| \leq 1$.

Received by the editors February 19, 2002 and, in revised form, March 26, 2003.

2000 Mathematics Subject Classification. Primary 47L70; Secondary 17C65.

Key words and phrases. JB* triple, bicircular projection, contractive projection, Hilbert space, Peirce decomposition. 
Example 1. Let $H$ be a complex Hilbert space and $a \in H$ a unit vector. Then the minimal projection, generated by $a$, is given by

$$
P x=\langle x, a\rangle a: x \in H .
$$

Obviously, $P$ has one-dimensional range. Moreover, for every real $\alpha$ and $\beta$ we have, since the ranges of $P$ and $\bar{P}$ are orthogonal,

$$
\begin{aligned}
\left\|e^{i \alpha} P x+e^{i \beta} \bar{P} x\right\|^{2} & =\|P x\|^{2}+\|\bar{P} x\|^{2} \\
& =\|\langle x, a\rangle x\|^{2}+\|x-\langle x, a\rangle x\|^{2}=\|x\|^{2}
\end{aligned}
$$

for all $x \in H$, which shows that $P$ is bicircular.

Example 2. Let $P: M_{2}(\mathbb{C}) \rightarrow M_{2}(\mathbb{C})$ be defined by

$$
P\left[\begin{array}{ll}
\alpha & \beta \\
\gamma & \delta
\end{array}\right]=\left[\begin{array}{ll}
\alpha & 0 \\
0 & \delta
\end{array}\right],
$$

and consider the matrix algebra equipped with the operator norm. Then, since

$$
\begin{aligned}
\left\|\left[\begin{array}{cc}
\alpha & \beta \\
\gamma & \delta
\end{array}\right]\right\| & \geq \max \{|\alpha|,|\beta|,|\gamma|,|\delta|\} \\
& \geq \max \{|\alpha|,|\delta|\}=\left\|\left[\begin{array}{cc}
\alpha & 0 \\
0 & \delta
\end{array}\right]\right\|,
\end{aligned}
$$

the projection $P$ is contractive. In a similar way we get that $\bar{P}$ is also contractive and so $P$ is bicontractive. We can see, however, that $P$ is not bicircular. If

$$
x=\left[\begin{array}{ll}
1 & 1 \\
1 & 1
\end{array}\right],
$$

then $\|x\|=2$, but for $\phi=P+i \bar{P}$ we have

$$
\phi x=\left[\begin{array}{ll}
1 & 0 \\
0 & 1
\end{array}\right]+\left[\begin{array}{ll}
0 & i \\
i & 0
\end{array}\right]=\left[\begin{array}{ll}
1 & i \\
i & 1
\end{array}\right] .
$$

Since $\|\phi x\|=\sqrt{2}$, the projection $P$ is not bicircular.

Historically $\mathrm{JB}^{*}$ triples arose in the study of bounded symmetric domains in complex holomorphy. They can be defined as those complex Banach spaces $W$ for which there exists a continuous triple product

$$
\{\ldots\}: W \times W \times W \rightarrow W
$$

such that

$\{x y z\}$ is symmetric and linear in $x, z$ and conjugate linear in $y$,

the linear operators $x \square x$, defined by $(x \square x)(y)=\{x x y\}$ are skew derivations of the operation $\{\ldots\}$ and they are Hermitian with nonnegative spectrum,

In particular, we have, by linearization of (JBii),

$$
\{a b\{x y z\}\}=\{\{a b x\} y z\}-\{x\{b a y\} z\}+\{x y\{a b z\}\} .
$$


Recall that by a skew derivation of $\{\ldots\}$ we mean a linear operator $D: W \rightarrow W$ such that

$$
D\{x y z\}=\{(D x) y z\}-\{x(D y) z\}+\{x y(D z)\}
$$

In the sequel we shall use the notation

$$
W(D, \alpha):=\{x \in W: D(x)=\alpha x\}
$$

for the eigensubspaces of skew derivations. Skew derivations have real spectrum and

$$
\{W(D, \alpha) W(D, \beta) W(D, \gamma)\} \subset W(D, \alpha-\beta+\gamma)
$$

Notice that a projection $P: W \rightarrow W$ is bicircular if and only if it is a skew derivation.

Indeed, if $P: W \rightarrow W$ is a projection, then $\exp ($ it $P)=e^{i t} P+\bar{P}$. If $P$ is bicircular, then these operators are surjective isometries for all real values of $t$. It is well known (see [12]) that surjective linear isometries and algebraic automorphisms are the same in $\mathrm{JB}^{*}$ triples. Furthermore, the infinitesimal generators of oneparameter groups of surjective linear isometries are exactly the derivations of the underlying triple product. Thus $i P$ is a derivation, and hence $P$ is a skew derivation of $\{\ldots\}$ for bicircular $P$. Conversely, if $P$ is supposed to be a skew derivation, then $\bar{P}$ is also a skew derivation and hence $i t P+i s \bar{P}$ is a derivation for any real $t$. It follows that $e^{i t} P+e^{i s} \bar{P}=\exp (i t P+i s \bar{P})$ is a surjective linear isometry for real $t$ and $s$.

This notion of $\mathrm{JB}^{*}$ triples can be regarded as a simultaneous generalization of Hilbert spaces and $\mathrm{C}^{*}$ algebras. If $H$ is a complex Hilbert space, we can define its JB* triple product by

$$
\{x y z\}=\frac{1}{2}\langle x, y\rangle z+\frac{1}{2}\langle z, y\rangle x
$$

If $A$ is a $\mathrm{C}^{*}$ algebra, we can define its $\mathrm{JB}^{*}$ triple product by

$$
\{x y z\}=\frac{1}{2} x y^{*} z+\frac{1}{2} z y^{*} x .
$$

$\mathrm{JB}^{*}$ triples were studied from algebraic (see for example [9, [10] and [14]), holomorphic (see for example [4], [11, [12] and [19]), operator-theoretic (see for example [7] and [15]) and Banach geometric (see for example [2], [3] and [5]) viewpoints. For a recent survey see [16].

In our first example we saw that every Hilbert space is a JB* triple with many rank one bicircular projections. This can easily be extended in the following way. Let $H$ be a complex Hilbert space and $P: H \rightarrow H$ a bicircular projection of rank one. Let $J$ be any $\mathrm{JB}^{*}$ triple and define

$$
Q: J \oplus H \rightarrow J \oplus H
$$

by $Q(j, h)=(0, P h)$. Then $Q$ is also a bicircular projection of rank one. Our main result shows that this is in fact the only possibility. More precisely, we shall prove

Theorem 1. Let $W$ be a $J B^{*}$ triple and $P: W \rightarrow W$ a bicircular projection of rank one. Then there exist two closed ideals $J$ and $H$ of $W$ such that $W=J \oplus H$, where $H$ is isomorphic to a Hilbert space, $P(W) \subset H$ and $J \subset \operatorname{ker}(P)$.

Corollary 1. The only prime JB* triples admitting bicircular projections of rank one are Hilbert spaces. 
Before we start our proof, we note that the assumption of rank one cannot be omitted from the above results. If $W=M_{2}(\mathbb{C})$, then $P: W \rightarrow W$, defined by

$$
P\left[\begin{array}{ll}
\alpha & \beta \\
\gamma & \delta
\end{array}\right]=\left[\begin{array}{ll}
\alpha & \beta \\
0 & 0
\end{array}\right]=\left[\begin{array}{ll}
1 & 0 \\
0 & 0
\end{array}\right]\left[\begin{array}{ll}
\alpha & \beta \\
\gamma & \delta
\end{array}\right],
$$

is bicircular, since for every real $t$ and $s$,

$$
\left(e^{i t} P+e^{i s} \bar{P}\right)\left[\begin{array}{cc}
\alpha & \beta \\
\gamma & \delta
\end{array}\right]=\left[\begin{array}{ll}
e^{i t} \alpha & e^{i t} \beta \\
e^{i s} \gamma & e^{i s} \delta
\end{array}\right]=\left[\begin{array}{cc}
e^{i t} & 0 \\
0 & e^{i s}
\end{array}\right]\left[\begin{array}{ll}
\alpha & \beta \\
\gamma & \delta
\end{array}\right]
$$

is a left multiplication with a unitary matrix.

\section{Proof of the main Result}

Since the range of $P$ is one-dimensional, we have $P(x)=f(x) a$ for some functional $f \in W^{\prime}$ and $a \in W$, both of norm one.

We divide the rest of the proof into several steps, to make its logic more clear.

Step 1. $P(W)=\mathbb{C} a$ where $a$ is an atom (also called minimal tripotent) of $W$. This means that $\{a a a\}=a$ and $\{a W a\}=\mathbb{C} a$.

Since $P^{2}=P$, we have $f(a)=1$. Since $P$ is a skew derivation,

$$
f(\{a a a\}) a=P\{a a a\}=\{P(a) a a\}-\{a P(a) a\}+\{a a P(a)\}=\{a a a\} .
$$

By the $\mathrm{C}^{*}$-equality (JBiii) in the definition of a $\mathrm{JB}^{*}$ triple, we have

$$
1=\|a\|^{3}=\|\{a a a\}\|=|f(\{a a a\})| \cdot\|a\|=|f(\{a a a\})| .
$$

Consequently, $\{a a a\}=e^{i \varphi} a$ for some real $\varphi$. From (JBii) it follows that

$$
e^{i \varphi} \in \sigma(a \square a) \subset \mathbb{R}^{+},
$$

and so $\{a a a\}=a$.

It remains to be proved that $\{a W a\}=\mathbb{C} a$. Actually, a stronger fact is true. Let $x \in W$ be arbitrary. Decompose

$$
x=P(x)+\bar{P}(x)=f(x) a+\bar{P}(x) .
$$

Since $P$ is a skew derivation with spectrum $\sigma(P)=\{0,1\}$, we have

$$
\{a \bar{P}(x) a\} \in\{W(P, 1) W(P, 0) W(P, 1)\} \subset W(P, 2)=0,
$$

and so

$$
\{a x a\}=\overline{f(x)}\{a a a\}+\{a \bar{P}(x) a\}=\overline{f(x)} a .
$$

Step 2. To every atom $a \in W$ there corresponds a so-called Peirce decomposition (cf. for example [14])

$$
W=W_{1} \oplus W_{\frac{1}{2}} \oplus W_{0}=\mathbb{C} a \oplus W_{\frac{1}{2}} \oplus W_{0}, \text { with }\left\{a W_{0} W\right\}=\left\{W_{0} a W\right\}=0,
$$

where $W_{\alpha}=\{x \in W:\{a a x\}=\alpha x\}=W(a \square a, \alpha)$. Now we claim that the Peirce projections $P_{\alpha}: W \rightarrow W_{\alpha}$ are skew derivations and $P_{1}=P$.

Before we proceed with our argument, we note that general Peirce projections are far from being skew derivations. By axiom (JBii), the operator $a \square a=$ $\sum_{\alpha=0,1 / 2,1} \alpha P_{\alpha}=P_{1}+\frac{1}{2} P_{\frac{1}{2}}$ is a skew derivation. Since $P$ is also a skew derivation, we have

$$
P(a \square a) x=P\{a a x\}=\{P(a) a x\}-\{a P(a) x\}+\{a a P(x)\}=(a \square a) P(x),
$$


that is, the operators $P$ and $a \square a$ commute and hence they leave the eigensubspaces of each other invariant. In particular,

$$
P W_{\alpha} \subset W_{\alpha} \cap P(W)=W_{\alpha} \cap \mathbb{C} a=0 \quad \text { for } \quad \alpha=0, \frac{1}{2} .
$$

Thus $W_{0} \oplus W_{\frac{1}{2}} \subset \operatorname{ker}(P)$. Since $P W=\mathbb{C} a=W_{1}$ and since $W=W_{1} \oplus W_{\frac{1}{2}} \oplus W_{0}$, it follows that $P_{1}=P$ is a skew derivation. Since real linear combinations of skew derivations are skew derivations, $P_{\frac{1}{2}}=2\left(P-P_{1}\right)$ and $P_{0}=\mathrm{Id}-P_{1}-P_{\frac{1}{2}}$ are skew derivations, too.

Step 3. We have $\left\{a W W_{0}\right\}=0$.

By the general rules of Peirce arithmetic we have

$$
\left\{a W_{0} W_{0}\right\} \subset\left\{W_{1} W_{0} W\right\}=0 \quad \text { and } \quad\left\{a W_{1} W_{0}\right\} \subset\left\{W_{0} W_{1} W\right\}=0 .
$$

So it remains to be proved that $\left\{a W_{\frac{1}{2}} W_{0}\right\}=0$. However, since $P_{\frac{1}{2}}$ is a skew derivation,

$$
\left\{a W_{\frac{1}{2}} W_{0}\right\} \subset\left\{W\left(P_{\frac{1}{2}}, 0\right) W\left(P_{\frac{1}{2}}, 1\right) W\left(P_{\frac{1}{2}}, 0\right)\right\} \subset W\left(P_{\frac{1}{2}},-1\right)=0 .
$$

Step 4. The subspaces $J=W_{0}$ and $H=\mathbb{C} a \oplus W_{\frac{1}{2}}$ are ideals of $W$ and so $W=J \oplus H$, where $P(J)=0$ and $a \in H$.

At this point we recall [2, Proposition 2.1], which states that given any sets $A, B \subset W$ with $\{A W B\}=0$, there exist two norm closed ideals $J, H$ of $W$, such that

$$
\begin{aligned}
J & =\{x \in W:\{A W x\}=0\} \supset B, \\
H & =\{x \in W:\{x W J\}=0\} \supset A, \text { and } \\
\{J W H\} & =J \cap H=0 .
\end{aligned}
$$

We use this for $A=\{a\}$ and $B=W_{0}$. Thus $W_{0} \subset J$. On the other hand, the ideal $H$ contains the atom $a$ and hence

$$
H \supset\{a W W\} \supset\{a a W\}=(a \square a) W=W_{1} \oplus W_{\frac{1}{2}} .
$$

Since $W=W_{1} \oplus W_{\frac{1}{2}} \oplus W_{0}$ and $\quad J \cap H=0$, necessarily $\quad J=W_{0}$ and $H=$ $W_{1} \oplus W_{\frac{1}{2}}$.

Now it only remains to prove that $H$ is isomorphic to a Hilbert space. In the special case of the so-called $\mathrm{JBW}^{*}$ triples it is a well-known classical result that an atom is a maximal tripotent in the same time iff the underlying space is Hilbert. We also feel that it should be known for the general case, but were not able to find such a statement in the literature. Since the argument is short, we include it for the sake of completeness.

Step 5. The ideal $H$ is isomorphic to a Hilbert space, with the triple product being the same as in the previous section.

It will be sufficient to construct an inner product on $W_{\frac{1}{2}}$. Take any $x, y$ in this subspace. Since

$$
\{a y x\} \in\left\{W_{1} W_{\frac{1}{2}} W_{\frac{1}{2}}\right\} \subset W_{1-\frac{1}{2}+\frac{1}{2}}=\mathbb{C} a,
$$

there exists a complex number $\langle x, y\rangle$ such that

$$
\{a y x\}=\frac{1}{2}\langle x, y\rangle a \text {. }
$$


From (JBi) it follows that this mapping is linear in $x$ and conjugate linear in $y$. Since

$$
\langle x, x\rangle \in \sigma(x \square x),
$$

the axiom (JBii) implies $\langle x, x\rangle \geq 0$.

If $\langle x, x\rangle=0$, we have $\{a x x\}=0$. Since, on the one hand,

$$
\{x a x\} \in\left\{W_{\frac{1}{2}} W_{1} W_{\frac{1}{2}}\right\} \subset W\left(a \square a, \frac{1}{2}-1+\frac{1}{2}\right)=W_{0}=J
$$

and on the other hand, since $H$ is an ideal of $W$, we have $\{x a x\} \in H$, it follows that $\{x a x\}=0$. Now we can use (JBii) to obtain

$$
-\frac{1}{2}\{x x x\}=-\left\{x\left(\frac{1}{2} x\right) x\right\}=-\{x\{x a a\} x\}=\{a x\{x a x\}\}-2\{\{a x x\} a x\}=0 .
$$

Using (JBiii), we get $x=0$, so that the mapping $x, y \longmapsto\langle x, y\rangle$ is in fact an inner product on $H$. Now it remains to verify the relation between this inner product and the triple product restricted to $W_{\frac{1}{2}}$. By linearization it suffices to prove that $\{x x x\}=\langle x, x\rangle x$ for $x \in W_{\frac{1}{2}}$.

Take any $x, w \in W_{\frac{1}{2}}$. Then by (JBii),

$$
\begin{aligned}
e & :=\langle\{x x x\}-\langle x, x\rangle x, w\rangle a \\
& =\langle\{x x x\}, w\rangle a-\langle x, x\rangle\langle x, w\rangle a \\
& =2\{\{x x x\} w a\}-4\{x x\{x w a\}\} \\
& =-2\{x\{w a x\} x\} .
\end{aligned}
$$

Since $\{\operatorname{wax}\} \in H \cap W_{0}=0, e=0$, and since $w$ was arbitrary, we obtain $\{x x x\}=$ $\langle x, x\rangle x$ as required. It now follows by (JBiii) that

$$
\|x\|^{2}=\langle x, x\rangle: x \in W_{\frac{1}{2}},
$$

which means that the norm of $W$, when restricted to $W_{\frac{1}{2}}$, is a pre-Hilbert norm. Since $W_{\frac{1}{2}}$ is closed in $W$, the completeness of $\left(W_{\frac{1}{2}},\langle.,\rangle.\right)$ also follows.

Now it is not difficult to verify that the inner product on $H$, given by

$$
\langle\alpha a+x, \beta a+y\rangle=\alpha \bar{\beta}+\langle x, y\rangle
$$

satisfies the same equalities. So, in fact, all of $H$ is a Hilbert triple.

With this, the proof of the main theorem is concluded. The corollary is now immediate. Since the ideal $H$ is always nonzero, its complement $J$ must be zero if $W$ is assumed to be prime. We conclude the paper with the following

Remark 1. The range of a contractive projection on a $\mathrm{JB}^{*}$-triple is again a $\mathrm{JB}^{*}$ triple in an abstract triple product ([6, [13] and [17]). If the projection is bicontractive, the range is a $\mathrm{JB}^{*}$-subtriple (see [8]).

Conjecture 1. Let $W$ be a $J B^{*}$ triple and $P: W \rightarrow W$ a nonzero bicircular projection whose range $P(W)$ is finite dimensional. Then $W$ is isomorphic to a direct sum of two ideals; one of them, containing $P(W)$, is either finite dimensional or isomorphic to a Hilbert triple. A prime JB*-triple with nonzero bicircular projection of finite rank is either finite dimensional or isomorphic to a Hilbert triple. 


\section{ACKNOWLEDGMENT}

The authors were supported by the Bilateral Slovenian-Hungarian Research Cooperation grant no. TET SLO-9/2000, OTKA T34267 of the Hungarian Scientific Research Foundation. They wish to express also their gratitude to the referee for his remarks which helped to improve the original manuscript considerably.

\section{REFERENCES}

[1] L. J. Bunce and C. H. Chu, Compact operations, multipliers and Radon-Nikodým property in JB* triples, Pacific J. Math. 153 (1992), 249-265. MR 93c:46125

[2] L. J. Bunce, C. H. Chu, L. L. Stachó and B. Zalar, On prime JB* triples, Quart. J. Math. Oxford 49 (1998), 279-290. MR 99j:46085

[3] C. H. Chu and P. Mellon, The Dunford-Pettis property in JB* triples, J. London Math. Soc. 55 (1997), 515-526. MR 98c:46146

[4] S. Dineen, The Schwarz Lemma, Oxford Mathematical Monographs, 1989. MR 91f:46064

[5] C. M. Edwards and G. T. Rüttimann, On the facial structure of the unit balls in a JBW* triple and its predual, J. London Math. Soc. 38 (1988), 317-332. MR 90b:46129

[6] Y. Friedmann and B. Russo, Solution of the contractive projection problem, J. Funct. Anal. 60 (1985), 56-79. MR 87a:46115

[7] Y. Friedmann and B. Russo, The Gel'fand-Nămark theorem for JB* triples, Duke Math. J. 53 (1986), 139-148. MR 87f:46086

[8] Y. Friedmann and B. Russo, Conditional expectation and bicontractive projections on Jordan $C^{*}$-algebras and their generalizations, Math. Z. 194 (1987), 227-236. MR 88c:46078

[9] G. Horn, Classification of $J B W^{*}$ triples of type I, Math. Z. 196 (1987), 271-291. MR 88m:46076

[10] G. Horn and E. Neher, Classification of continuous JBW* triples, Trans. Amer. Math. Soc. 306 (1988), 553-578. MR 89c:46090

[11] J. Isidro and W. Kaup, Determining boundary sets of bounded symmetric domains, Manuscripta Math. 81 (1993), 149-159. MR 94i:32054

[12] W. Kaup, A Riemann mapping theorem for bounded symmetric domains in complex Banach spaces, Math. Z. 183 (1983), 503-529. MR 85c:46040

[13] W. Kaup, Contractive projections on Jordan $C^{*}$-algebras and generalizations, Math. Scand. 54 (1984), 95-100. MR 85h:17012

[14] O. Loos, Jordan Pairs, Lecture Notes in Mathematics, vol. 460, Springer-Verlag, 1975. MR 56:3071

[15] A. Rodriguez, On the strong* topology of a JBW* triple, Quart. J. Math. Oxford 42 (1991), 99-103. MR 92j:46123

[16] B. Russo, Structure of JB* triples, in Jordan Algebras (eds. W. Kaup, K. McCrimmon and H. P. Petersson), de Gruyter, Berlin, 1994. MR 95h:46109

[17] L. L. Stachó, A projection principle concerning biholomorphic automorphisms, Acta Sci. Math. (Szeged) 44 (1982), 99-124. MR 83j:32034

[18] L. L. Stachó and B. Zalar, Symmetric continuous Reinhardt domains, Arch. Math. (Basel) 81 (2003), 50-61.

[19] H. Upmeier, Symmetric Banach manifolds and Jordan $C^{*}$-algebras, North-Holland, Amsterdam, 1985. MR 87a:58022

University of Szeged, Bolyai Institute, Aradi Vértanúk tere 1, 6720 Szeged, Hungary

E-mail address: stacho@math.u-szeged.hu

URL: http://www.math.u-szeged.hu

University of Maribor, Smetanova 17, 2000 Maribor, Slovenia

E-mail address: borut.zalar@uni-mb.si 